\title{
Variants in SBF2 gene associated with survival in pancreatic adenocarcinoma
}

A two-stage genome-wide association study (GWAS) published in Gut has demonstrated that genetic variation in $S B F 2$ is associated with overall survival in patients with pancreatic adenocarcinoma.

Patients with pancreatic cancer have very poor 5-year survival rates of around 5\%. Although GWASs have identified genetic variants associated with disease development, few of these types of studies have investigated associations with overall survival of patients.

$\mathrm{Wu}$ and colleagues analysed genetic variants associated with overall survival in $>1,000$ patients with pancreatic adenocarcinoma. The first stage of the study analysed genetic data from 642 patients of European descent. The top 131 single nucleotide polymorphisms (SNPs) from this analysis were then advanced to a joint analysis that included a further 362 patients of

Chinese ancestry.

The second stage of the analysis revealed that the SNP most highly associated with overall survival in the joint population was rs 10500715 , which is located in the SBF2 gene on chromosome 11p15.4. This SNP was associated with a hazard ratio for death of 0.76 .

The authors conclude that this association should be investigated further in larger cohorts of patients. Improved understanding of the effect of these variants could help in defining patient prognosis and the biology of pancreatic cancer progression.

Isobel Franks

Original article Wu, C. et al. Genome-wide association study of survival in patients with pancreatic adenocarcinoma. Gut doi:10.1136/gutjnl-2012-303477 